\title{
Genomic and metabolic insights into Thermoanaerobacterium thermosaccharolyticum GSU5 solvent production
}

\author{
Rocio Díaz Peña ${ }^{1}$, Daniela Alvarez ${ }^{1}$, Diego Egoburo ${ }^{1}$, Jimena Ruiz ${ }^{1}$, and Julia Pettinari ${ }^{1}$ \\ ${ }^{1}$ University of Buenos Aires
}

April 28, 2020

\begin{abstract}
Thermoanaerobacterium thermosaccharolyticum GSU5 was isolated from animal dung collected in a pasture plain in Buenos Aires, Argentina. This thermophilic and anaerobic microorganism was able to produce butanol and ethanol, but not acetone, using sugars such as xylose, arabinose, glucose, galactose, fructose, sucrose and cellobiose. Key metabolic enzymes leading to solvent production were identified in its genome. A detailed analysis of the solvent and organic acid biosynthetic pathway genes of sequenced strains revealed new insights into the unique metabolic features of this species. The large range of fermentable substrates and the ability to produce both ethanol and butanol without acetone makes this species an interesting candidate for second generation biofuel production.
\end{abstract}

\section{Introduction}

Climate change is a threat to the several life forms of our planet. This phenomenon is partially due to the preferential use of fossil fuels to sustain human activities such as heating, agriculture or transportation. The use of biofuels is one of the most promising alternatives, as it would help reduce the effects of climate change, while avoiding the use of non-renewable resources such as petroleum or gas as sources of energy (Hill, Nelson, Tilman, Polasky, \& Douglas, 2006). Among the most common biofuels are alcohols that can be obtained from microbial fermentation using different kinds of carbon sources. Ethanol is the best known and most studied alcohol that can be obtained by microbial fermentation using yeast or bacteria, while butanol is a less common but increasingly attractive biofuel due to several characteristics, since it is capable of producing more energy if properly harnessed, and has a lower vapor pressure and is less hygroscopic than ethanol (Dürre, 2007). Many different processes have been developed to obtain alcohols from sugars or starch, but the use of these substrates to produce biofuels would compete with food supplies. To avoid this problem, biofuels should be obtained from non-food substrates, such as lignocellulosic biomass. Several different approaches have been employed to use this abundant substrate, most of which start with the hydrolysis of the biomass to obtain sugars that can be subsequently fermented to the desired compounds. This hydrolysis is normally achieved through energy intensive processes, involving high temperatures and aggressive chemical conditions. Consolidated bioprocessing is an alternative that integrates enzyme production, saccharification and fermentation (Yamada, Hasunuma, \& Kondo, 2013) that can be achieved in mild conditions. Consolidated bioprocesses that use anaerobic thermophilic organisms capable of degrading lignocellulosic biomass are expected to meet sustainability standards, as they would enable production of biofuels from renewable resources by means of low energy demanding procedures (Lynd, Weimer, Zyl, \& Pretorius, 2002).

The use of cellulolytic thermophilic bacteria, such as C. thermocellum, has been extensively studied for the production of ethanol from cellulose (Lamed \& Zeikus, 1980; Tian et al., 2016). However, most of these organisms are unable to produce butanol, and/or to ferment pentoses derived from hemicellulose degradation, thereby limiting the efficient use of lignocellulosic biomass (Demain, Newcomb, \& Wu, 2005). In recent years, several species of the thermophilic genusThermoanaerobacterium have received increased attention due to 
their capability to use different biomass substrates to produce solvents. The genus Thermoanaerobacterium comprises 8 validly described species: T . aciditolerans, T. aotearoense, T. saccharolyticum, T. thermosaccharolyticum, T. thermostercoris (formerly T. thermostercus), T. thermosulfurigenes, T. xylanolyticumand T. butyriciformans (Onyenwoke \& Wiegel, 2015). T. saccharolyticum and T. thermostercoris can produce ethanol (Romano et al., 2010; Shaw et al., 2008) and several T. thermosaccharolyticum isolates have been reported to produce hydrogen (Cao et al., 2009) and/or butanol (Li, Zhang, Yang, \& He, 2018) from different biomass sources. These results point out the potential of these microorganisms for the synthesis of diverse bioproducts, and particularly biofuels, from untreated or minimally treated biomass, and spur further research in order to analyze their potential application in consolidated bioprocesses.

We have isolated and characterized a new strain, T. thermosaccharolyticum GSU5, an anaerobic thermophilic bacterium that is capable of producing ethanol and butanol from a variety of substrates. In this work we present the genomic sequence of GSU5 and analyze its phenotypic traits, especially those pertaining to solvent production, in comparison to the type strain of the species. Additionally, genes related to solventogenesis of all sequenced Thermoanaerobacteriumare compared to gain new insights into their unique metabolic properties.

\section{Materials and methods}

\section{Bacterial strains and growth conditions}

Thermoanaerobacterium thermosaccharolyticum GSU5 was isolated from animal dung collected in a pasture plain in Buenos Aires, Argentina, in 1987. The strain was originally designated Clostridium thermopapyrolyticum due to its phenotypic characteristics (Mendez, Pettinari, Ivanier, Ramos, \& Siñeriz, 1991). Stock cultures were kept at $4^{\circ} \mathrm{C}$ in Hungate tubes containing growth medium with a strip of filter paper for several decades. Thermoanaerobacterium thermosaccharolyticum DSM 571 was obtained from Deutsche Sammlung von Mikroorganismen und Zellkulturen (DSMZ, Germany).

Both strains were grown at $60{ }^{\circ} \mathrm{C}$ in $5 \mathrm{ml}$ screw cap tubes using the Hungate method (Hungate, 1950) in TSC medium (Shaw et al., 2012) containing per liter: $2.0 \mathrm{~g}$ sodium citrate tribasic dihydrate, $1.85 \mathrm{~g}$ of (NH4)2SO4, 0.1 g FeSO4.7H2O, 2.0 g MgSO4.7H2O, 1.0 g KH2PO4, 0.1 g CaCl2.2H2O, resazurin 2 mg 8.5 $\mathrm{g}$ yeast extract, $10 \mathrm{~g}$ glucose. The $\mathrm{pH}$ was adjusted to 6.7 with $\mathrm{NaOH} 10 \mathrm{M}$.

For metabolite production analysis, the strains were grown in TSC medium supplemented with $10 \mathrm{~g} / \mathrm{L}$ of different carbon sources: monosaccharides xylose, arabinose, glucose, galactose and fructose; and disaccharides sucrose and cellobiose. The $\mathrm{pH}$ was adjusted to 6.7 with $\mathrm{NaOH} 10 \mathrm{M}$. The strains were grown at $60^{\circ} \mathrm{C}$ for 48 hours.

Fermentations were performed in a 2.5 L BiostatA bioreactor with 1.5 L of TSC supplemented with 10g/L of carbon sources (glucose or xylose). After $17 \mathrm{hs} 5 \mathrm{~g} / \mathrm{L}$ of the corresponding sugar were added to avoid carbon source depletion. The agitation was kept at $100 \mathrm{rpm}$. The $\mathrm{pH}$ was adjusted initially at 7 and was regulated with $\mathrm{NaOH} 2 \mathrm{M}$ and $\mathrm{H} 2 \mathrm{SO} 41 \mathrm{M}$ to keep it over 5. TSC pre-cultures were grown in serum bottles containing $50 \mathrm{ml}$ of medium at anaerobic conditions, $150 \mathrm{ml}$ of inoculum was added to the bioreactor $(\mathrm{DO}=0,3)$. The strains were grown at $60^{\circ} \mathrm{C}$ for 35 hours.

\section{Sequence analysis}

The sequence was obtained using a whole-genome shotgun strategy with a Roche 454 GS FLX Titanium pyrosequencer at INDEAR, Argentina, achieving 37-fold coverage. Assembly was done using Newbler version 2.6 and generated 73 contigs, the largest of which has 81,110 bases. The annotation was performed using the NCBI Prokaryotic Genomes Automatic Annotation Pipeline (PGAAP) and Rapid Annotations using Subsystems Technology (RAST) (Overbeek et al., 2014). The genome of GSU5 was deposited in Genbank with the accession number MINB01000001-MINB01000073.

Genome to genome distance calculations were performed using the GGDC program (https://ggdc.dsmz.de/), that provides an in silico estimation of DNA-DNA hybridization values (Auch, Klenk, \& Göker, 2010) 
and Average Nucleotide Identity based on blast (ANIb) http://jspecies.ribohost.com/ jspeciesws (Richter, Rosselló-Móra, Oliver Glöckner, \& Peplies, 2016).

\section{Analytical determinations}

Cultures were centrifuged for 5 minutes at $6000 \mathrm{rpm}$, the supernatants were filtered through $0.22 \mu \mathrm{m}$ nylon membranes (MSI, USA) and stored at $-20{ }^{\circ} \mathrm{C}$ for analytical determinations.

Organic acids were determined by high-performance liquid chromatography (HPLC) using an LC-20AT Prominance equipment (Shimadzu Corp., Kyoto, Japan) with an HPX-87-H Aminex column (Cat no. 125170 0140; Bio Rad Laboratories Inc., Hercules, CA) at $50^{\circ} \mathrm{C}$. The mobile phased consisted of $5 \mathrm{mM}$ sulfuric acid at a flow rate of $0,6 \mathrm{ml} / \mathrm{min}$. The metabolites were quantified with a SPD-20AV UV detector (Shimadzu 171 Corp.).

Ethanol and butanol were measured by Manual Head Space GC-FID using an Agilent 7820A GC-FID with manual head space injection. The separation was conducted on a HP-INNO- WAX capillary column $(30 \mathrm{~m}$, $0.25 \mu \mathrm{m}$ film thickness and $0.25 \mathrm{~mm}$ ID). The supernatants were diluted $\frac{1}{2}$ in $\mathrm{K} 2 \mathrm{CO} 3 \mathrm{~g} / \mathrm{ml}$, and $5 \mu \mathrm{l}$ of isobutanol $5 \mathrm{~g} / \mathrm{L}$ was added as an internal standard. The samples were heated at $60^{\circ} \mathrm{C}$ for $1 \mathrm{~h}$ and $1 \mathrm{ml}$ of the gas phase was injected manually in a GC-FID. The GC oven was initially heated at $35{ }^{\circ} \mathrm{C}$ for 2 min, then to $45{ }^{\circ} \mathrm{C}$ at $30^{\circ} \mathrm{C} / \mathrm{min}$ and held for $1 \mathrm{~min}$, finally, was heated to $120^{\circ} \mathrm{C}$ at $50^{\circ} \mathrm{C} / \mathrm{ml}$ and maintained for 1 min. The injector and FID temperatures were set at 150 and $300^{\circ} \mathrm{C}$, respectively. Nitrogen was used as the carrier gas at a column flow of $2 \mathrm{ml} / \mathrm{min}$ with a 5:1 split ratio.

\section{Results}

\section{Genomic relatedness of $T$. thermosaccharolyticum GSU5}

The draft genome sequence of T. thermosaccharolyticum GSU5 was obtained in 73 contigs with a total length of 2.7 kbases, with a mean $\mathrm{G}+\mathrm{C}$ content of $33.9 \%$. Analysis of the genome using the NCBI Prokaryotic Genomes Automatic Annotation Pipeline (PGAAP) revealed 2668 genes, comprising 2501 predicted coding regions, 102 pseudogenes and 65 RNA genes, including one 16S rRNA gene. The RAST annotation covered 339 subsystems, including $44 \%$ of the coding sequences, and classified 897 open reading frames (ORFs) as hypothetical proteins.

Comparison of the 16S rRNA gene of GSU5 against sequences deposited in the Ribosomal Database Project revealed that GSU5 clusters together with other strains of Thermoanaerobacterium thermosaccharolyticum and is closest to strain TG57 (Figure 1).

The overall genomic phylogenetic relatedness of strain GSU5 with other strains of the genus Thermoanaerobacterium was analyzed throughin silico genome comparisons. Values obtained by means of in silico determination of genome to genome distances between GSU5 and all other available Thermoanaerobacterium genomes corroborate that it belongs to the T. thermosaccharolyticumspecies. Values of $87.30 \%$, $76.40 \%, 75.40 \%$ and $72.10 \%$ were obtained when GSU5 was compared with T. thermosaccharolyticum TG57, T.thermosaccharolyticum DSM 571, T. thermosaccharolyticumM5, and T. thermosaccharolyticum M0795 respectively, while the values obtained with all other strains ranged between 26.40 and 24.20\% (Table 1). When Average Nucleotide Identity based on BLAST (ANIb) was calculated, the values obtained (96.28 98.04\%) when GSU5 was compared with other T. thermosaccharolyticum strains provided further evidence that it belongs to this species (Table 1).

Among the 11 Thermoanaerobacterium genomes available, the best represented species is T. thermosaccharolyticum, with five sequenced strains, followed by T. saccharolyticum, with two strains, and a single representative each for T. xylanolyticumand T. aotearoense. The other two genomes correspond to strains RBIITD and PSU2. When the genome of Thermoanaerobacterium sp. RBIITD was compared against all strains of the genus Thermoanaerobacterium DDH values ranged between 21.3 and $24.70 \%$ and ANI values ranged between 75.92 and $79.93 \%$ (Table S1). Similar results were obtained for Thermoanaerobacterium sp. PSU2, with DDH values ranging from 21.30 and $58.30 \%$ and ANI values between 76.68 and $94.20 \%$ 
(Table S2). These results indicate that these strains do not belong to any of the species with sequenced representatives: T. thermosaccharolyticum, T. saccharolyticum, T. xylanolyticum or T. aotearoense .

\section{Solvent synthesis pathways}

The key enzymes involved in the synthesis of butanol, ethanol and acetone were searched in the genome of GSU5 and other sequenced Thermoanaerobacterium strains using the RAST annotation Server, BLAST and the Biocyc database collection in order to analyze the corresponding pathways.

As shown in Table 2, all genes coding for key enzymes of butanol synthesis were detected in GSU5 and all sequenced T. thermosaccharolyticum strains (locus tags in Table S3). Genes coding for the crotonase ( crt ), butyryl-CoA dehydrogenase ( $b c d$ ), electron transfer flavoprotein (etfAB ), 3-hydroxybutyryl-CoA dehydrogenase ( $h b d)$, and acetyl-CoA C-acetyltransferase $(t h l)$, are located in a cluster, followed by but , that codes for a butyryl-CoA: acetate-CoA transferase, and rex, corresponding to a redox dependent transcriptional regulator (Figure 2). In the well-known butanol producer Clostridium acetobutylicumseven of the genes are also in a genomic cluster, the bcs operon, albeit with a different organization: in this organism thl is located in a different region, rex is situated upstream fromcrt, and but is absent.

Putative binding sites for the transcriptional regulator Rex (ROP: Rex operator site) were observed upstream from thl (Figure 2) and adhE in the genome of GSU5, suggesting that Rex could regulate the expression of these genes in Thermoanaerobacterium . Butanol synthesis genes were also found in Thermoanaerobacterium $s p$. RBIITD but with a genetic organization different from the one present in T. thermosaccharolyticum, since thl is located in the bcsoperon but rex is located upstream from crt (Figure 2). In contrast, these genes were absent in T. xylanolyticum LX-11, T. saccharolyticum JW/SL-YS485, T. saccharolyticum NTOU1, T. aotearoense SCUT27 and Thermoanaerobacterium sp. PSU-2, which suggests that they are not capable of producing butanol (Table 2).

There are 5 genes encoding alcohol dehydrogenases distributed throughout the genome of GSU5 and one encoding an aldehyde dehydrogenase (ald ). This last gene was present in about half of the analyzed genomes, including three out of five sequenced T. thermosaccharolyticum (Table 2). All Thermoanaerobacteriumstrains carry adhE, coding for the bifunctional alcohol/aldehyde dehydrogenase, in a monocistronic operon with a similar location in all genomes analyzed, as well as bhd and two other alcohol dehydrogenases. Interestingly, one of the dehydrogenases, adh3, was located upstream from genes coding for a NADH-dependent reduced ferredoxin: NADP oxidoreductase $(n f n A B)$ in all genomes.

The well characterized C. acetobutylicum produces butanol and acetone through the ABE (Acetone-ButanolEthanol) pathway, so the possibility that T. thermosaccharolyticum produced this compound was studied. The genes that code for acetone synthesis enzymes were searched in the genomes of $T$. thermosaccharolyticum GSU5 and in all available Thermoanaerobacterium genomes using the genes from $C$. acetobutylicum as queries. None of the genomes analyzed carried $c \operatorname{tf} A B$ or $a d c$, the genes that code for the butyrate-acetoacetate CoA-transferase (subunits A and B), and the acetoacetate decarboxylase respectively (Table 2).

Genes corresponding to enzymes involved in the synthesis of acetate and butyrate (Table 2) were also identified. T. thermosaccharolyticumGSU5 presents the gene coding for butyryl-CoA: acetate CoA- transferase (But) which catalyzes the conversion of butyryl-CoA to butyrate, located downstream from the butanol synthesis genes. This gene was also found in all other strains of this species, and in Thermoanaerobacteriumsp. RBIITD, albeit in this last case but is located in a different part of the genome (Figure 2). On the other hand T. xylanolyticum LX-11, T. saccharolyticum JW/SL-YS485, T. saccharolyticum NTOU1, T. aotearoense SCUT27 and T. sp.PSU-2 carry the genes coding for Ptb (that catalyzes the synthesis of butyryl-CoA to butyryl-P), and Buk (that catalyzes the conversion of butyryl-P to butyrate).

\section{Production of metabolites from different carbon sources}

The synthesis of solvents and acids by T. thermosaccharolyticumGSU5 was analyzed in cultures grown using different substrates in $5 \mathrm{ml}$ tubes, and compared with the type strain of the species, DSM 571 . Both strains grew well on several monosaccharides: glucose, xylose, arabinose and galactose, and also on the disaccharides 
sucrose and cellobiose. They grew slightly more when using fructose that on pentoses and other hexoses, and also showed a modest preference for cellobiose compared to sucrose (despite their specific epithet). In all cases they produced alcohols and acids, but no acetone. Ethanol was the most abundant alcohol, and butanol was also observed in all cultures in lower amounts. Among the acids, butyrate was the most abundant, followed by acetate, with lower amounts of lactate (Figure 3).

The relative production of the metabolites depended on the substrate used, and some differences were observed between the strains. The main product of GSU5 was butyrate in all conditions tested, ranging from 0.93 to $2.07 \mathrm{~g} / \mathrm{L}$, while DSM 571 synthesized butyrate as a main product $(0.99$ to $1.35 \mathrm{~g} / \mathrm{L})$ in all cultures except those using glucose and xylose, in which similar amounts of ethanol and butyrate were observed (Figure 3). Both strains produced much more ethanol than butanol. GSU5 produced between 0.12 and 0.73 $\mathrm{g} / \mathrm{L}$ ethanol and 0.005 to $0.048 \mathrm{~g} / \mathrm{L}$ butanol, with the highest amount of butanol produced in sucrose. DSM 571 produced between 0.25 and $1.25 \mathrm{~g} / \mathrm{L}$ ethanol and 0.014 to $0.034 \mathrm{~g} / \mathrm{L}$ butanol with the highest amount of butanol obtained in glucose.

Strain GSU5 produced 3.2 times more butanol than DSM 571 in sucrose $(0.048$ vs $0.015 \mathrm{~g} / \mathrm{L} ; \mathrm{p}<0.05)$, and also accumulated more butanol than DSM571 in arabinose, while the opposite was observed in fructose and cellobiose, in which strain DSM571 produced more butanol than GSU5. When ethanol production was compared, strain DSM 571 produced a greater concentration of ethanol than GSU5 in all hexoses and in xylose, in which the highest amount was observed $(0.98 \mathrm{~g} / \mathrm{L})$, while GSU5 accumulated more ethanol in arabinose and with the disaccharides sucrose and cellobiose, with the maximum production in this last carbon source $(0.73 \mathrm{~g} / \mathrm{L})$.

\section{Bioreactor cultures}

Strain GSU5 was grown in bioreactor batch cultures using glucose or xylose as carbon sources in order to analyze solvent production in a larger scale. Several tests were performed to determine the best $\mathrm{pH}$ conditions for growth and solvent production, since previous studies had indicated that solvent production was enhanced by low $\mathrm{pH}$ in other T. thermosaccharolyticum strains. Preliminary tests in glucose cultures with initial starting $\mathrm{pH}$ of 6.7 or 7.5 did not allow adequate growth, so all cultures were performed with an initial $\mathrm{pH}$ of 7 , and $\mathrm{pH}$ was controlled to prevent values lower than 5. Sugars were added at an initial concentration of $10 \mathrm{~g} / \mathrm{L}$, and replenished after $17 \mathrm{~h}$ by adding half the initial amount to avoid sugar depletion.

Growth was more vigorous in glucose cultures, achieving $52 \%$ more biomass than when using xylose (1.9 vs $1.0 \mathrm{~g} / \mathrm{L})$. Solvent production was also much higher in glucose, while xylose cultures produced more acids than solvents (Figure 4). Butyric acid remained the main product in xylose cultures, but glucose cultures produced slightly more ethanol than butyrate. The final butanol concentration obtained was $0.33 \pm 0.01$ $\mathrm{g} / \mathrm{L}$ in glucose and $0.26 \pm 0.02 \mathrm{~g} / \mathrm{L}$ in xylose, while the final ethanol concentration was $4.34 \pm 0.06 \mathrm{~g} / \mathrm{L}$ in glucose and $0.78 \pm 0.49 \mathrm{~g} / \mathrm{L}$ in xylose (Table 3 ).

As expected, fermentor cultures corresponding to both sugars had a much higher biomass and solvent production that $5 \mathrm{ml}$ tube cultures. Although the main products were the same observed in tube cultures, the relative amounts of acids and alcohols varied. A higher relative amount of butanol was observed with both substrates when all major metabolites were considered, showing a slight increase in carbon fluxes towards butanol in bioreactor cultures (Figure 5). When cultures grown using glucose were compared to tube cultures, solvent production was observed to increase more than biomass, with the most important increase observed in butanol production. While an 8 fold change was observed in biomass (1.78 vs $0.22 \mathrm{~g} / \mathrm{L})$, final concentrations of ethanol and butanol increased 10 and 14 times respectively, so that the amount of alcohols produced in the fermentor was similar to that of acids (Figure 5). Results obtained with xylose were different, as a 6 fold increase in biomass $(1.04$ vs $0.20 \mathrm{~g} / \mathrm{L})$ was accompanied by a similar increase in butanol concentration, while ethanol concentration only increased 1.5 fold when fermentor cultures were compared with $5 \mathrm{ml}$ cultures (Figure 5).

\section{Discussion}


In this work we describe a novel strain of Thermoanaerobacterium thermosaccharolyticum (strain GSU5) through genome analysis and metabolic characterization. The phylogenetic analysis revealed that strain GSU5 belongs to the T. thermosaccharolyticum species and is closest to strain TG57. A genomic analysis of all sequenced strains of Thermoanaerobacterium was also performed, including two strains that have not yet been assigned to any species, PSU2 and RBIITD. When strain PSU2 was first described, it was classified as a strain of T. thermosaccharolyticum (O-Thong, Prasertsan, Karakashev, \& Angelidaki, 2008), and when its genome was published, the authors reported that according to in silico studies, it did not belong to the $T$. xylanolyticum species (O-Thong et al., 2017). The results presented in this work indicate that neither PSU2 nor RBIITD belong to T. saccharolyticum, T . thermosaccharolyticum, T. xylanolyticum or T. aotearoense . It remains to be studied whether these strains belong to species with no sequenced representatives, or to previously undescribed Thermoanaerobacterium species.

T. thermosaccharolyticum synthesizes several products of biotechnological importance, among which ethanol and butanol are the most relevant, along with hydrogen (Bhandiwad, Guseva, \& Lynd, 2013). Strains GSU5 and DSM 571 produced both alcohols from all carbon sources tested in this study, including glucose, fructose, galactose, xylose, arabinose, cellobiose and sucrose. The organization of genes involved in butanol synthesis is the same in both strains, and different from the organization of the well-known bcs operon found in solventogenic Clostridium (Berezina, Brandt, Yarotsky, Schwarz, \& Zverlov, 2009) in which the thl gene is not part of the bcs operon, but constitutes a monocistronic operon in another part of the genome (Wietzke \& Bahl, 2012).

It is known that in C. acetobutylicum the transcriptional regulator Rex plays a fundamental role in the regulation of solvent synthesis (Panitz et al., 2014) and binds to specific sequences identified upstream from thl, crt(the first gene of the bcs operon), and adhE (Wietzke \& Bahl, 2012). A gene coding for Rex was identified downstream from thebsc operon in T. thermosaccharolyticum GSU5 and DSM571, and putative Rex binding sequences (ROP) were found both upstream fromthl (the first gene of the bsc operon) and of adhE , suggesting that Rex could regulate the expression of these genes, as described in Clostridium . Butanol synthesis has been reported previously not only in several strains of $T$. thermosaccharolyticum but also in Thermoanaerobacterium sp. RBIITD (Biswas et al., 2018). In this last organism, the structure of the $b c s$ operon is the same as in T. thermosaccharolyticum, except that rex is upstream from the bcs operon, as in C. acetobutylicum .

All genomes analyzed carry adhE, coding for the bifunctional alcohol/aldehyde dehydrogenase, while ald , corresponding to an aldehyde dehydrogenase, was only found in GSU5 and in some butanol producing and non-butanol producing strains. T. thermosaccharolyticum DSM 571 and M5 do not have genes coding for this enzyme, but produce both butanol and ethanol (Bhandiwad et al., 2013; Jiang et al., 2018; Li et al., 2018). These results suggest thatald is not essential for alcohol synthesis in Thermoanaerobacterium, in agreement with previous reports that indicated that $\mathrm{AdhE}$ was responsible for the synthesis of n-butanol from butyryl-coA and ethanol from acetyl-coA (Bhandiwad et al., 2014). All strains also carried genes coding for other dehydrogenases, including Bhd, so a possible role of these enzymes in the synthesis of alcohols in Thermoanaerobacterium cannot be ruled out.

When T. thermosaccharolyticum GSU5 or DSM 571 were grown in different carbon sources, several of the typical metabolites associated to butanol producers were detected, such as butyrate, acetate, lactate, ethanol and butanol, but no acetone could be found in any of the cultures. In addition, it has been reported that butanol producing strains RBIITD and TG57 do not produce acetone during fermentation (Biswas et al., 2018; Li et al., 2018). A search of the genome of GSU5 for the presence of genes related to acetone synthesis revealed that it lacks $a d c$, coding for an acetate decarboxylase, and $\operatorname{ctf} A B$, which codes for both units of a butyrate-acetoacetate CoA-transferase (Figure 6).

In recent years analysis of the genome of several strains of $T$. thermosaccharolyticum revealed important differences in the biosynthesis of butanol and butyrate when compared to the pathway known in the well characterized C. acetobutylicum (Jones \& Woods, 1986). Previous reports indicated that adc was absent in T. thermosaccharolyticum M5 (Jiang et al., 2018), and that bothadc and ctf were absent in T. thermo- 
saccharolyticumTG57 ( $\mathrm{Li}$ et al., 2018). The analysis performed in this work revealed a general absence of these genes in all the genomes, indicating that none of the strains analyzed would be able to produce acetone during fermentation due to the absence of $a d c$ and $c t f$. Since the analysis involved all available Thermoanaerobacterium genomes representing half of the known species, these results suggest that this is a common trait in this genus.

Butyrate production has been reported for several T. thermosaccharolyticum strains (Biswas et al., 2018; Freier-Schröder, Wiegel, \& Gottschalk, 1989; Li et al., 2018), and is the main metabolite produced from most carbon sources by both strains tested in this work. Among the known butyrate producing pathways, the most common is the acetyl-CoA pathway, that has two variants: i) the two step conversion catalyzed by the phosphotransbutyrylase (Ptb) and butyrate kinase (Buk) with a phosphorylated intermediate that allows the formation of ATP, commonly found in bacteria that have the ABE pathway, and ii) the one step conversion of Butyryl-CoA to Butyrate catalyzed by a butyryl-CoA:acetate-CoA transferase (But) (Vital, Howe, \& Tiedje, 2014). Only the one step pathway seems to be performed by strain GSU5 and all other T. thermosaccharolyticum strains, as they carrybut (denominated ach in T. thermosaccharolyticumTG57), and not $p t b$ or buk (Bhandiwad et al., 2013; Li et al., 2018) (Figure 6). The two step pathway has been proposed to exist in strain M5 (Jiang et al., 2018) but its genome does not carry genes coding for Ptb or Buk.

The one step conversion of butyryl-CoA to butyrate has been extensively studied in C. kluyveri, that seems to compensate the lack of the ATP producing step by means of an electron bifurcating mechanism involving the crotonyl-CoA reductase, that couples the reduction of crotonyl-CoA to the reduction of ferredoxin using $\mathrm{NADH}$ as the electron donor for both reactions. The reduction of crotonyl-CoA to butyryl-CoA is catalyzed by the cytoplasmic butyryl-CoA dehydrogenase complex, coded by $b c d$ and etf $A B$, while the reduction of the ferredoxin is catalyzed by NfnAB, a NADH-dependent reduced ferredoxin:NADP oxidoreductase (Wang, Huang, Moll, \& Thauer, 2010). These genes are present in all the butanol and butyrate producing strains: T. thermosaccharolyticum and Thermoanaerobacterium sp. RBIITD (table 2). Furthermore, the ferredoxin dependent activity of the butyryl-CoA dehydrogenase was experimentally demonstrated in $T$. thermoanaerobacterium DSM 571 (Bhandiwad et al., 2014). Based on this information, it can be hypothesized that all butanol producing Thermoanaerobacterium are able to obtain energy during the one step butyrate synthesis through the ferredoxin mediated electron bifurcation mechanism (Figure 6). In contrast, genes coding for Ptb and Buk were identified in T. xylanolyticum, T. saccharolyticum, T. aotearoense and Thermoanaerobacteriumsp. PSU-2. These strains lack genes needed for butanol synthesis (bcs operon), and are also devoid of genes coding for the cytoplasmic butyryl-CoA dehydrogenase complex (bcd andetfAB), suggesting that they are unable to synthesize either butanol or butyrate. In these microorganisms pbt and bukare clustered together with a gene that codes for a leucine/valine/phenylalanine dehydrogenase. This genetic organization has been previously observed in Bacillus megaterium. In this organism Ptb expression was induced in the presence of valine and isoleucine, and the enzyme could use butyryl-CoA and 2-methylpropionyl CoA as substrates (Vazquez, Pettinari, \& Méndez, 2001). In B. subtilis these genes are part of the $b k d$ operon, involved in the degradation of branched chain amino-acids (Debarbouille, Gardan, Arnaud, \& Rapoport, 1999). It is possible that in T. xylanolyticum ,T. saccharolyticum, Thermoanaerobacterium sp. PSU-2 and T. aotearoense Ptb and Buk could be involved in branched chain amino-acid degradation as in Bacillus .

Strain GSU5 was able to simultaneously produce both organic acids and alcohols in different carbon sources in a similar manner as the control strain DSM 571. The main acid produced was butyrate, the most abundant metabolic product in most conditions. Both strains were able to synthesize ethanol and smaller amounts of butanol from all carbon sources tested. These results are similar to those obtained in previous studies performed using T. thermosaccharolyticum DSM 571 grown on cellobiose (Bhandiwad et al., 2013), and also using strain M5 grown on xylan (Jiang et al., 2018). In contrast, Li et al report that T. thermosaccharolyticum TG57 produce butyrate, acetate and butanol, but no ethanol, when grown using glucose, cellobiose, cellulose or xylan, in spite of the fact that it has all enzymes required for the synthesis of both alcohols (Li et al., 2018). 
When GSU5 was grown in bioreactors, it achieved a higher biomass, and some differences were observed in the relative amounts of acids and alcohols produced. For example, while butyrate was the most abundant metabolite produced in $5 \mathrm{ml}$ tube cultures using all substrates tested, including glucose and xylose, fermentor cultures with those substrates had a greater proportion of alcohols. Furthermore, ethanol was the most abundant metabolite in glucose cultures. This could suggest that in higher density cultures, carbon flow towards the synthesis of alcohols increases contributing to the reoxidation of excess NADH or NADPH, thus favoring the production of alcohols over acids. Solventogenic anaerobes like the broadly studied model bacterium C. acetobutylicum have a fermentation behavior characterized by two distinct phases: formation of acids during the first phase is followed by a solventogenic phase in which growth slows down, and solvents are produced (Amador-Noguez, Brasg, Feng, Roquet, \& Rabinowitz, 2011). When the dynamics of acids and alcohols production was analyzed in T. thermosaccharolyticumGSU5, metabolite synthesis was observed to accompany growth, and no clear solventogenic phase could be distinguished. This had been reported for T. thermosaccharolyticum M5 (Jiang et al., 2018) and careful observation of metabolite curves displayed in studies carried out with different solventogenic Thermoanaerobacterium strains show that in all cases alcohols and acids are synthesized throughout growth. This can be clearly perceived in an early work performed using strain DSM 571 (Freier-Schröder et al., 1989) and in the metabolite curves shown in studies that analyze the production of hydrogen in different strains of T. thermosaccharolyticum (Cao et al., 2009; Cao, Zhao, Wang, Wang, \& Ren, 2014; Khamtib \& Reungsang, 2012), using both sugars or lignocellulosic biomass for growth. These results suggest that solventogenesis in Thermoanaerobacterium is not subject to the same regulatory mechanisms described for the ABE metabolism in

\section{Clostridium.}

Several species of Thermoanaerobacterium have been studied for the production of different bioproducts. Among them, T. thermosaccharolyticum seems to be the most promising, and different strains have been analyzed as candidates for the production of hydrogen (Cao et al., 2009, 2014), ethanol (Bhandiwad et al., 2013, 2014; Jiang, Guo, et al., 2018; Li et al., 2018) and 1,2 propanediol (Altaras, Etzel, \& Cameron, 2001; Cameron \& Cooney, 1986) from several carbon sources, including lignocellulosic biomass. Knowledge on their physiology and unique metabolic properties will enable the use of these microorganisms to obtain biotechnologically relevant bioproducts in sustainable and environmentally friendly processes.

\section{Acknowledgements}

We wish to thank Dr. Beatriz Méndez for her expert advice and Dr. Laura Raiger Iustman for allowing us to use the bioreactor. M.J.P. and J.R. are career investigators from Consejo Nacional de Investigaciones Científicas y Técnicas (CONICET, Argentina). R.D.P., D.E.E., and D.S.A. hold doctoral fellowships from CONICET.

\section{Conflicts of interest .}

Authors declare no conflict of interest.

\section{References}

Altaras, N. E., Etzel, M. R., \& Cameron, D. C. (2001). Conversion of sugars to 1,2-propanediol by Thermoanaerobacterium thermosaccharolyticum HG-8. Biotechnology Progress ,17 (1), 52-56. https://doi.org/10.1021/bp000130b

Amador-Noguez, D., Brasg, I. A., Feng, X. J., Roquet, N., \& Rabinowitz, J. D. (2011). Metabolome remodeling during the acidogenic-solventogenic transition in Clostridium acetobutylicum. Applied and Environmental Microbiology , 77 (22), 7984-7997. https://doi.org/10.1128/AEM.05374-11

Auch, A. F., Klenk, H.-P., \& Göker, M. (2010). Standard operating procedure for calculating genome-togenome distances based on high-scoring segment pairs. Standards in Genomic Sciences ,2 (1), 142-148. https://doi.org/10.4056/sigs.541628

Berezina, O. V., Brandt, A., Yarotsky, S., Schwarz, W. H., \& Zverlov, V. V. (2009). Isolation of a new butanol-producing Clostridiumstrain: High level of hemicellulosic activity and structure of solventogenesis 
genes of a new Clostridium saccharobutylicumisolate. Systematic and Applied Microbiology, 32 (7), 449-459. https://doi.org/10.1016/j.syapm.2009.07.005

Bhandiwad, A., Guseva, A., \& Lynd, L. R. (2013). Metabolic Engineering of Thermoanaerobacterium thermosaccharolyticum for Increased n- Butanol Production for n -butanol production. Advances in Microbiology , 2013 (August 2015), 46-51. https://doi.org/10.1016/j.ymben.2013.10.012

Bhandiwad, A., Shaw, A. J., Guss, A., Guseva, A., Bahl, H., \& Lynd, L. R. (2014). Metabolic engineering of Thermoanaerobacterium saccharolyticum for n -butanol production. Metabolic Engineering , 21 , 17-25. https://doi.org/10.1016/j.ymben.2013.10.012

Biswas, R., Huntemann, M., Clum, A., Pillay, M., Palaniappan, K., Varghese, N., .. Gussc, A. M. (2018). Complete Genome Sequence of Thermoanaerobacterium sp. Strain RBIITD, a Butyrate- and Butanol-Producing Thermophile Ranjita. Genome Announcements ,6 (2), e01411-17.

Cameron, D. C., \& Cooney, C. L. (1986). A novel ferementation: The production of R(-)-1,2-propanediol and acetol by Clostridium Thermosaccharolyticum . Nature Biotechnology , 4 (August), 719-725.

Cao, G., Ren, N., Wang, A., Lee, D. J., Guo, W., Liu, B., ... Zhao, Q. (2009). Acid hydrolysis of corn stover for biohydrogen production using Thermoanaerobacterium thermosaccharolyticum W16.International Journal of Hydrogen Energy , 34 (17), 7182-7188. https://doi.org/10.1016/j.ijhydene.2009.07.009

Cao, G., Zhao, L., Wang, A. J., Wang, Z. Y., \& Ren, N. Q. (2014). Single-step bioconversion of lignocellulose to hydrogen using novel moderately thermophilic bacteria. Biotechnology for Biofuels ,7 (1), 1-13. https://doi.org/10.1186/1754-6834-7-82

Debarbouille, M., Gardan, R., Arnaud, M., \& Rapoport, G. (1999). Role of bkdR, a transcriptional activator of the SigL-dependent isoleucine and valine degradation pathway in Bacillus subtilis . Journal of Bacteriology , 181 (7), 2059-2066.

Demain, A. L., Newcomb, M., \& Wu, J. H. D. (2005). Cellulase, Clostridia, and Ethanol. Thermophysics and Aeromechanics ,69 (1), 124-154. https://doi.org/10.1128/MMBR.69.1.124-154.2005

Dürre, P. (2007). Biobutanol: An attractive biofuel. Biotechnology Journal , 2 (12), 1525-1534. https://doi.org/10.1002/biot.20

Freier-Schröder, D., Wiegel, J. irge., \& Gottschalk, G. (1989). Butanol formation by Clostridium thermosaccharolyticum at neutral pH Doris, 11 (11), 831-836.

Hill, J., Nelson, E., Tilman, D., Polasky, S., \& Douglas, T. (2006). Environmental, economic, and energetic costs and benefits of biodiesel and ethanol biofuels. Proceedings of the National Academy of Sciences , 103 (30), 11206-11210. https://doi.org/10.5506/APhysPolB.44.1379

Hungate, R. E. (1950). The Anaerobic Mesophilic Cellulolytic Bacteria.Bacteriological Reviews , 14, 1-49.

Jiang, Y., Guo, D., Lu, J., Dürre, P., Dong, W., Yan, W., ... Xin, F. (2018). Consolidated bioprocessing of butanol production from xylan by a thermophilic and butanologenic Thermoanaerobacterium sp. M5.Biotechnology for Biofuels , 11 (1), 1-14. https://doi.org/10.1186/s13068-018-1092-1

Jones, D. T., \& Woods, D. R. (1986). Acetone-Butanol Fermentation Revisited. Microbiological Reviews, 50 (4), 484-524.

Khamtib, S., \& Reungsang, A. (2012). Biohydrogen production from xylose by Thermoanaerobacterium thermosaccharolyticum KKU19 isolated from hot spring sediment. International Journal of Hydrogen Energy ,37 (17), 12219-12228. https://doi.org/10.1016/j.ijhydene.2012.06.038

Lamed, R., \& Zeikus, J. G. (1980). Ethanol production by thermophilic bacteria: relationship between fermentation product yields of and catabolic enzyme activities in Clostridium thermocellum and Thermoanaerobium brockii . Journal of Bacteriology ,144 (2), 569-578. https://doi.org/10.1038/ng1296-461 
Li, T., Zhang, C., Yang, K. L., \& He, J. (2018). Unique genetic cassettes in a Thermoanaerobacterium contribute to simultaneous conversion of cellulose and monosugars into butanol. Science Advances , 4 (3). https://doi.org/10.1126/sciadv.1701475

Lynd, L. R., Weimer, P. J., Zyl, W. H. van, \& Pretorius, I. S. (2002). Microbial Cellulose Utilization: Fundamentals and Biotechnology.Microbiology and Molecular Biology Reviews , 63 (3), 506-577. https://doi.org/10.1128/MMBR.66

Mendez, B. S., Pettinari, M. J., Ivanier, S. E., Ramos, C. A., \& Siñeriz, F. (1991). Clostridium thermopapyrolyticum sp. nov., a cellulolytic thermophile. International Journal of Systematic Bacteriology , 281-283. https://doi.org/10.1099/00207713-41-2-281

O-Thong, S., Khongkliang, P., Mamimin, C., Singkhala, A., Prasertsan, P., \& Birkeland, N. K. (2017). Draft genome sequence of Thermoanaerobacterium sp. strain PSU-2 isolated from thermophilic hydrogen producing reactor. Genomics Data , 12 , 49-51. https://doi.org/10.1016/j.gdata.2017.02.012

O-Thong, S., Prasertsan, P., Karakashev, D., \& Angelidaki, I. (2008). Thermophilic fermentative hydrogen production by the newly isolated Thermoanaerobacterium thermosaccharolyticum PSU-2.International Journal of Hydrogen Energy , 33 (4), 1204-1214. https://doi.org/10.1016/j.ijhydene.2007.12.015

Onyenwoke, R. U., \& Wiegel, J. (2015). Thermoanaerobacterium .Bergey's Manual of Systematics of Archaea and Bacteria , 1-18. https://doi.org/10.1002/9781118960608.gbm00755

Overbeek, R., Olson, R., Pusch, G. D., Olsen, G. J., Davis, J. J., Disz, T., .. Stevens, R. (2014). The SEED and the Rapid Annotation of microbial genomes using Subsystems Technology (RAST). Nucleic Acids Research , 42 (D1), 206-214. https://doi.org/10.1093/nar/gkt1226

Panitz, J. C., Zverlov, V. V., Pham, V. T. T., Stürzl, S., Schieder, D., \& Schwarz, W. H. (2014). Isolation of a solventogenic Clostridium sp. strain: Fermentation of glycerol to n-butanol, analysis of the bcs operon region and its potential regulatory elements. Systematic and Applied Microbiology , 37 (1), 1-9. https://doi.org/10.1016/j.syapm.2013.10.004

Richter, M., Rosselló-Móra, R., Oliver Glöckner, F., \& Peplies, J. (2016). JSpeciesWS: A web server for prokaryotic species circumscription based on pairwise genome comparison. Bioinformatics ,32 (6), 929-931. https://doi.org/10.1093/bioinformatics/btv681

Romano, I., Dipasquale, L., Orlando, P., Lama, L., d'Ippolito, G., Pascual, J., \& Gambacorta, A. (2010). Thermoanaerobacterium thermostercus sp. nov., a new anaerobic thermophilic hydrogen-producing bacterium from buffalo-dung. Extremophiles , 14 (2), 233-240. https://doi.org/10.1007/s00792-010-0303-x

Shaw, A. J., Podkaminer, K. K., Desai, S. G., Bardsley, J. S., Rogers, S. R., Thorne, P. G., .. Lynd, L. R. (2008). Metabolic engineering of a thermophilic bacterium to produce ethanol at high yield.Proceedings of the National Academy of Sciences , 105 (37), 13769-13774. https://doi.org/10.1073/pnas.0801266105

Shaw, J. A., Covalla, S. F., Miller, B. B., Firliet, B. T., Hogsett, D. A., \& Herring, C. D. (2012). Urease expression in a Thermoanaerobacterium saccharolyticum ethanologen allows high titer ethanol production. Metabolic Engineering , 14 (5), 528-532. https://doi.org/10.1016/j.ymben.2012.06.004

Tian, L., Papanek, B., Olson, D. G., Rydzak, T., Holwerda, E. K., Zheng, T., ... Lynd, L. R. (2016). Simultaneous achievement of high ethanol yield and titer in Clostridium thermocellum .Biotechnology for Biofuels , 9 (1), 1-11. https://doi.org/10.1186/s13068-016-0528-8

Vazquez, G. J., Pettinari, M. J., \& Méndez, B. S. (2001). Phosphotransbutyrylase expression in Bacillus megaterium .Current Microbiology , 42 (5), 345-349. https://doi.org/10.1007/s002840010227

Vital, M., Howe, A. C., \& Tiedje, J. M. (2014). Revealing the bacterial butyrate synthesis pathways by analyzing (meta)genomic data.MBio , 5 (2), 1-11. https://doi.org/10.1128/mBio.00889-14

Wang, S., Huang, H., Moll, J., \& Thauer, R. K. (2010). NADP+ reduction with reduced ferredoxin and $\mathrm{NADP}+$ reduction with NADH are coupled via an electron-bifurcating enzyme complex in Clostridium 
kluyveri .Journal of Bacteriology , 192 (19), 5115-5123. https://doi.org/10.1128/JB.00612-10

Wietzke, M., \& Bahl, H. (2012). The redox-sensing protein Rex, a transcriptional regulator of solventogenesis in Clostridium acetobutylicum . Applied Microbiology and Biotechnology, 96 (3), 749-761. https://doi.org/10.1007/s00253012-4112-2

Yamada, R., Hasunuma, T., \& Kondo, A. (2013). Endowing non-cellulolytic microorganisms with cellulolytic activity aiming for consolidated bioprocessing. Biotechnology Advances , 31 (6), 754-763. https://doi.org/10.1016/j.biotechadv.2013.02.007

Table 1: Genome to genome comparisons of GSU5 with other Thermoanaerobacterium .

\begin{tabular}{llll}
\hline Strain & Accession & DDH estimate & ANIb \\
T. thermosaccharolyticum GSU5 & MINB01000001-MINB01000073 & - & \\
T. thermosaccharolyticum TG57 & CP016893.1 & $87.30 \pm 2.50 \%$ & $98.04 \%$ \\
T. thermosaccharolyticum DSM 571 & CP002171 & $76.40 \pm 2.88 \%$ & $96.48 \%$ \\
T.thermosaccharolyticum M5 & NKHD01000001-NKHD01000077 & $75.40 \pm 3.00 \%$ & $96.93 \%$ \\
T. thermosaccharolyticum M0795 & NC_019970 & $72.10 \pm 2.92 \%$ & $96.28 \%$ \\
T. saccharolyticum JW/SL-YS485 & CP003184.1 & $24.30 \pm 2.39 \%$ & $81.63 \%$ \\
T. saccharolyticum NTOU1 & BBKT01000001-BBKT01000101 & $26.40 \pm 2.50 \%$ & $83.52 \%$ \\
T. xylanolyticum LX-11 & CP002739.1 & $25.00 \pm 2.40 \%$ & $82.12 \%$ \\
T. aotearoense SCUT27 & AYSN01000000 & $24.20 \pm 2.50 \%$ & $81.76 \%$ \\
Thermoanaerobacterium sp. PSU-2 & MSQD00000000.1 & $24.40 \pm 2.50 \%$ & $81.82 \%$ \\
Thermoanaerobacterium sp. RBIITD & LT906662.1 & $24.30 \pm 2.50 \%$ & $79.52 \%$ \\
\hline
\end{tabular}

DNA-DNA hybridization (DDH) values were estimated using the Genome-to-Genome Distance Calculator 2.1 (Species cutoff value: 70\%). ANIb: Average Nucleotide Identity based on BLAST (Species cutoff value $=95 \%$ ).

Table 2. Genes involved in ethanol, butanol and butyrate synthesis in sequenced strains

\begin{tabular}{|c|c|c|c|c|c|c|c|c|c|c|c|c|}
\hline Strain & crt & $b c d$ & etf $B$ & etf $A$ & $h b d$ & thl & but & $p t b$ & buk & ald & $a d h 1$ & adh2 \\
\hline T. thermosaccharolyticum GSU5 & 100 & 100 & 100 & 100 & 100 & 100 & 100 & & & 100 & 100 & 100 \\
\hline T. thermosaccharolyticum TG57 & 100 & 100 & 99 & 100 & 100 & 100 & 100 & & & 58 & 100 & 99 \\
\hline T. thermosaccharolyticum DSM 571 & 91 & 98 & 99 & 99 & 100 & 100 & 100 & & & & 99 & 99 \\
\hline T. thermosaccharolyticum M5 & 99 & 99 & 99 & 99 & 100 & 100 & 100 & & & & 99 & 100 \\
\hline T. thermosaccharolyticum M0795 & 99 & 99 & 98 & 95 & 98 & 100 & 100 & & & 58 & & 99 \\
\hline T. saccharolyticum JW/SL-YS485 & & & & & & & & 0 & 0 & 93 & & 92 \\
\hline T. saccharolyticum NTOU1 & & & & & & & & 0 & 0 & & 98 & 92 \\
\hline T. xylanolyticum LX-11 & & & & & & & & 0 & 0 & 92 & & 92 \\
\hline T.aotearoense SCUT27 & & & & & & & & 0 & 0 & 93 & & 92 \\
\hline Thermoanaerobacterium sp. PSU-2 & & & & & & & & 0 & 0 & & & 91 \\
\hline Thermoanaerobacterium sp. RBIITD & 77 & 96 & 91 & 96 & 88 & 90 & 82 & 0 & 0 & 58 & 67 & 89 \\
\hline Clostridium acetobutylicum ATCC 824 & 58 & 72 & 66 & 58 & 69 & 71 & & 0 & 0 & & & \\
\hline
\end{tabular}

Numbers indicate percentage of protein sequence identity with the corresponding protein of strain GSU5. Red cells indicate that the gene is present in the corresponding strain and absent in GSU5. Blank cells indicate absence of the gene in the corresponding strain. Genes code for the proteins indicated as follows, crt: crotonase; $b c d$ : butyryl-CoA dehydrogenase; etfAB: electron transfer flavoprotein; $h b d: 3$-hydroxybutyrylCoA dehydrogenase; thl : acetyl-CoA C-acetyltransferase; but : butyryl-CoA: acetate-CoA transferase; $p t b$ : phosphotrans butyrylase; buk : butyrate kinase; ald : aldehyde dehydrogenase; $a d h$ : alcohol dehydrogenase; $b d h$ : butanol dehydrogenase; adhE : bifunctional aldehyde/ alcohol dehydrogenase; pta : phosphotrans 
acetylase; $a k$ : acetate kinase; $c t f A B$ : acetoacetyl coenzyme A:acetate/butyrate:coenzyme A transferase; $a d c$ : acetoacetate decarboxylase. Accession numbers for each genome are indicated in table 1.

Table 3. Fermentation products of Thermoanaerobacterium thermosaccharolyticum GSU5

\begin{tabular}{|c|c|c|c|c|c|c|c|c|}
\hline & $\begin{array}{l}\text { Product } \\
\text { concentra- } \\
\text { tion } \\
\mathrm{g} / \mathrm{L}\end{array}$ & $\begin{array}{l}\text { Product } \\
\text { concentra- } \\
\text { tion } \\
\mathrm{g} / \mathrm{L}\end{array}$ & Yield a g/g & Yield a g/g & $\begin{array}{l}\text { Yieldb mol } \\
/ \text { mol }\end{array}$ & $\begin{array}{l}\text { Yieldb mol } \\
/ \mathrm{mol}\end{array}$ & $\begin{array}{l}\text { Volumetric } \\
\text { productivi- } \\
\text { tyc } \\
\mathrm{g} /(\mathrm{L} \cdot \mathrm{h})\end{array}$ & $\begin{array}{l}\text { Volur } \\
\text { prode } \\
\text { tyc } \\
\mathrm{g} /(\mathrm{L} .\end{array}$ \\
\hline & Glu & Xyl & Glu & $\mathrm{Xyl}$ & Glu & $\mathrm{Xyl}$ & Glu & Xyl \\
\hline Butanol & $0.33 \pm 0.10$ & $0.26 \pm 0.15$ & $0.034 \pm 0.008$ & $0.022 \pm 0.007$ & $0.09 \pm 0.02$ & $0.05 \pm 0.01$ & $0.015 \pm 0.004$ & 0.013 \\
\hline Ethanol & $4.35 \pm 0.06$ & $0.8 \pm 0.5$ & $0.46 \pm 0.07$ & $0.059 \pm 0.04$ & $1.8 \pm 0.1$ & 0.23 & $0.19 \pm 0.01$ & $.03=$ \\
\hline Butyrate & $3 \pm 1$ & $6 \pm 3$ & $39 \pm$ & $0.33 \pm 0.19$ & $0.8 \pm 0.3$ & $0.6 \pm 0.3$ & $0.12 \pm 0.08$ & $.28=$ \\
\hline Acetate & $2.3 \pm 0.9$ & $0.96 \pm 0.2$ & $0.13 \pm 0.08$ & $0.10 \pm 0.07$ & $0.5 \pm 0.2$ & $0.15 \pm 0.07$ & $0.11 \pm 0.04$ & $.05=$ \\
\hline Lactate & $1.1 \pm 0.3$ & $1.2 \pm 0.8$ & $0.15 \pm 0.07$ & $0.09 \pm 0.07$ & $0.22 \pm 0.06$ & $0.2 \pm 0.1$ & $0.05 \pm 0.01$ & \\
\hline Biomass & $1.9 \pm 0.7$ & $1.0 \pm 0.5$ & $0.19 \pm 0.09$ & $0.09 \pm 0.05$ & - & - & - & 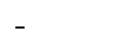 \\
\hline
\end{tabular}

Cultures were grown in TSC medium supplemented with glucose or xylose at 10/L in a bioreactor under anaerobic conditions for $36 \mathrm{~h}$. Results represent means \pm the standard deviations corresponding to three independent cultures. Glu, Glucose; Xyl, xylose.

a Yield: $g$ of product/ $g$ of substrate

b yield: : mol of product/mol of substrate

c: g of product/ (time . volume )

\section{Figure Legends}

Figure 1: Relatedness of GSU5 with other bacteria.

The 16s rRNA gene of GSU5 was compared against the sequences deposited in the Ribosomal Database Project. A phylogenetic tree was obtained using the Mega 6.0 program. At least one representative of each species of Thermoanaerobacterium, and all strains with sequenced genomes were included, along with the two species of Caldanaerobium, formerly classified within the Thermoanaerobacterium genus.Clostridium acetobutylicum was used as an outgroup.

*Strains with sequenced genomes

Figure 2: Organization of the bcs operon in Thermoanaerobacterium thermosaccharolyticum, Thermoanaerobacterium sp. RBIITD and Clostridium acetobutylicum. Putative Rex operator sites (ROP) are indicated as red circles.

Figure 3: Production of metabolites in T. thermosaccharolyticumGSU5 (A) and T. thermosaccharolyticum DSM 571 (B). Strains were grown in TCS supplemented with different carbon sources in 5 ml Hungate tubes under anaerobic conditions for $48 \mathrm{~h}$. Results represent the mean value \pm standard deviation of three independent replicates.

Figure 4: Production of metabolites in T. thermosaccharolyticumGSU5 grown in TCS supplemented with glucose (A) or xylose (B) at $10 \mathrm{~g} / \mathrm{L}$ in a bioreactor under anaerobic conditions for $36 \mathrm{~h}$.

Data from a representative fermentation of three independent replicates is presented.

Figure 5: Relative distribution (\%) of metabolites produced by T. thermosaccharolyticum GSU5 in Hungate tubes with glucose (A), in a bioreactor with glucose (B), in Hungate tubes with xylose (C), and in a bioreactor with xylose (D).

Figure 6: Proposed main metabolic pathways in T. thermosaccharolyticum. 
Purple solid arrows indicate reactions catalyzed by genes present in the genome. Red dotted arrows indicate reactions catalyzed by genes absent in the genome. Enzymes separated by a slash and/or with asterisks indicate reactions that could be potentially catalyzed by more than one enzyme. Enzyme names are: Crt: crotonase; Bcd: butyryl-CoA dehydrogenase; EtfAB: electron transfer flavoprotein; Hbd: 3-hydroxybutyrylCoA dehydrogenase; Thl: acetyl-CoA C-acetyltransferase; But: butyryl-CoA: acetate-CoA transferase; Ptb: phosphotrans butyrylase; Buk: butyrate kinase; Ald: aldehyde dehydrogenase; Bdh: butanol dehydrogenase; AdhE: bifunctional aldehyde/ alcohol dehydrogenase; Pta: phosphotrans acetylase; Ak: acetate kinase; CtfAB: acetoacetyl coenzyme A:acetate/butyrate:coenzyme A transferase; Adc: acetoacetate decarboxylase; Fd red: reduced ferredoxin; Fd ox: oxidized ferredoxin.

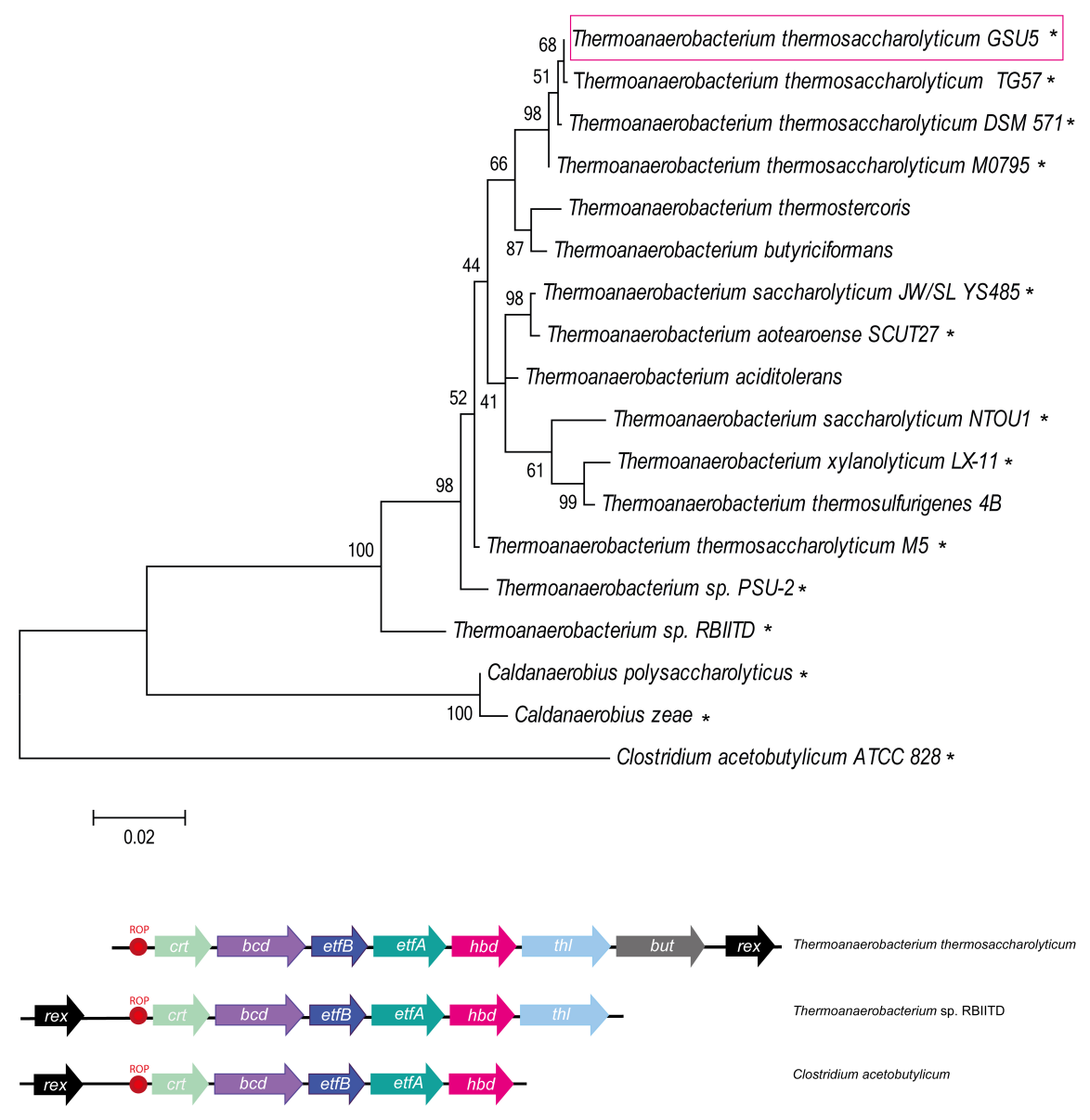



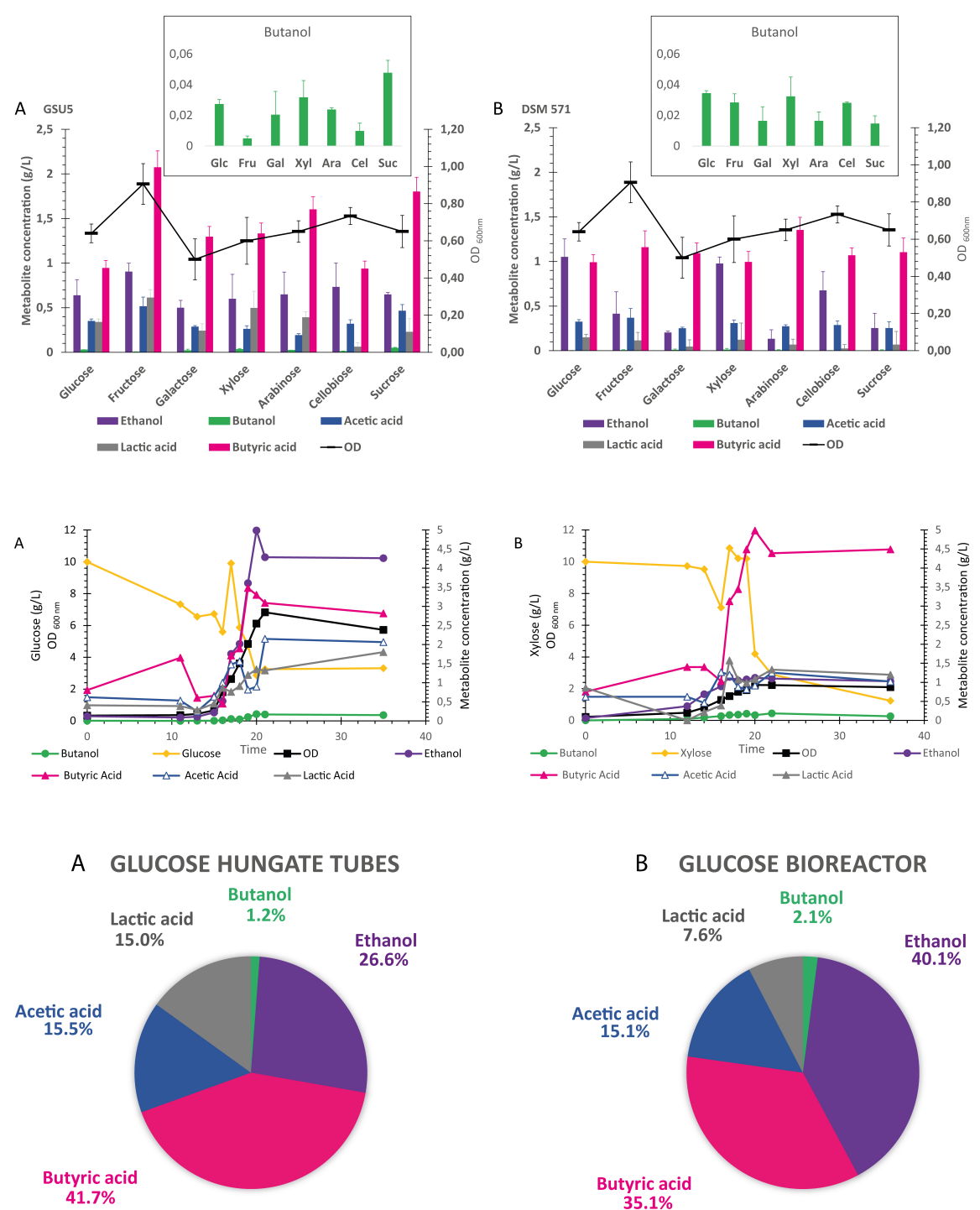

B GLUCOSE BIOREACTOR

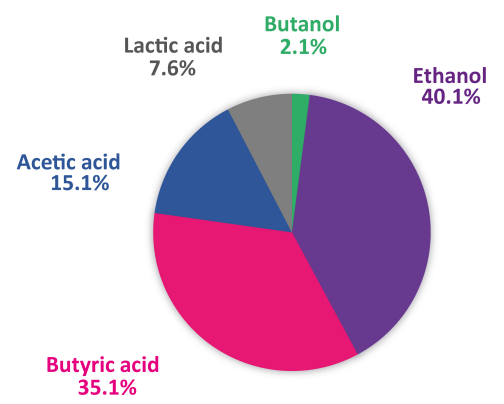

C XYLOSE HUNGATE TUBES
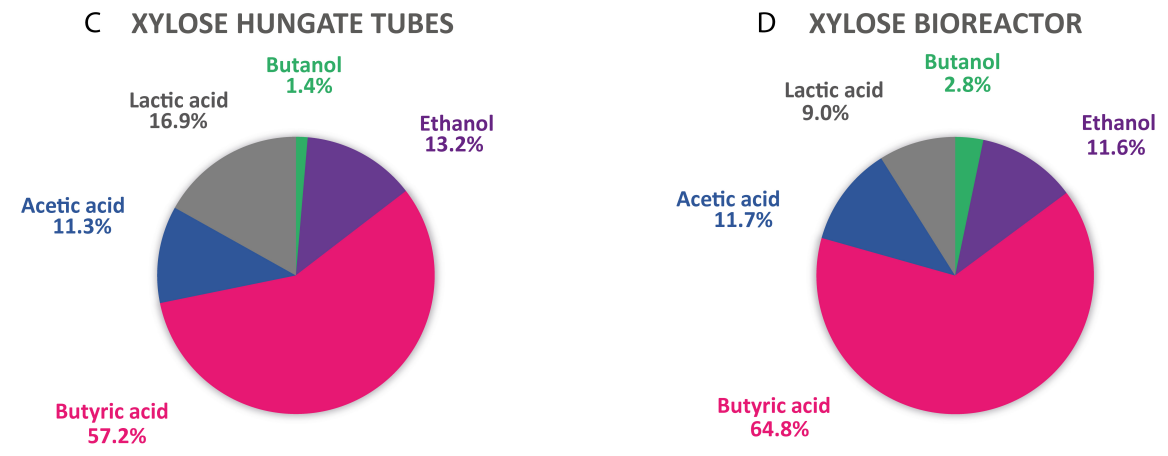


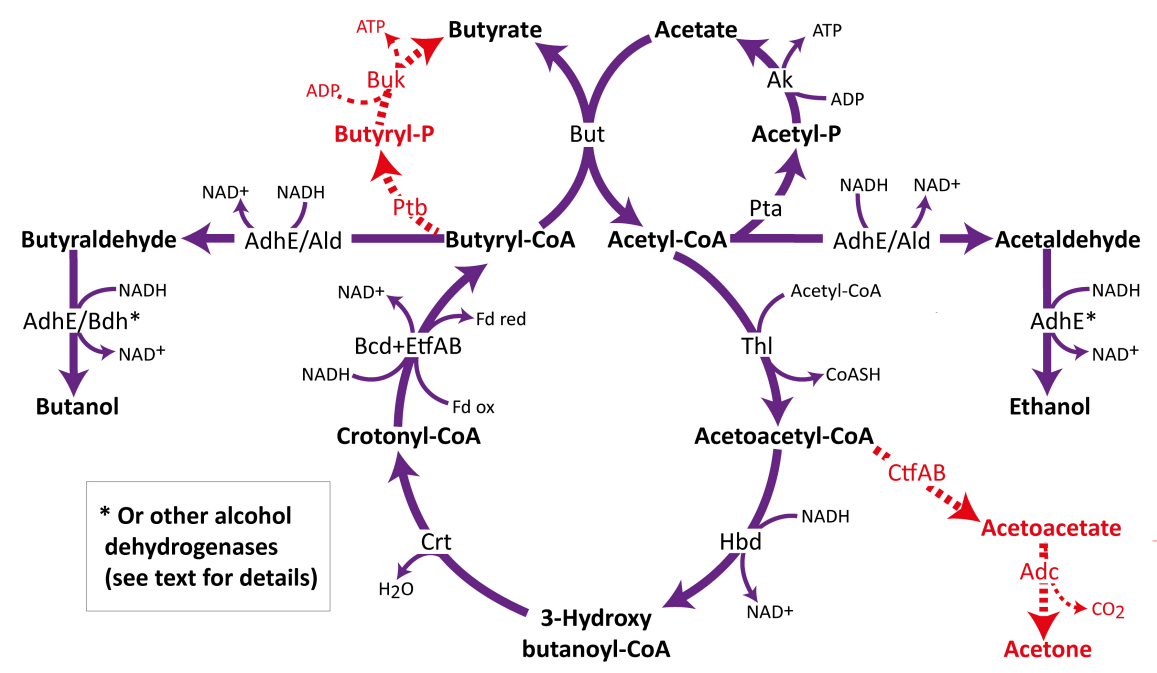

